\title{
School performance in children with infantile hydrocephalus: a nationwide cohort study
}

This article was published in the following Dove Press journal:

Clinical Epidemiology

\author{
Linnea Boegeskov Schmidt ${ }^{1}$ \\ Giulia Corn' \\ Jan Wohlfahrt' \\ Mads Melbye ${ }^{1-3}$ \\ Tina Noergaard Munch ${ }^{1,4}$ \\ 'Department of Epidemiology \\ Research, Statens Serum Institut, \\ Copenhagen, Denmark; ${ }^{2}$ Department \\ of Clinical Medicine, University of \\ Copenhagen, Copenhagen, Denmark; \\ ${ }^{3}$ Department of Medicine, Stanford \\ University School of Medicine, \\ Stanford, CA, USA; ${ }^{4}$ Department of \\ Neurosurgery, Copenhagen University \\ Hospital, Copenhagen, Denmark
}

Correspondence: Linnea Boegeskov Schmidt

Department of Epidemiology Research, Statens Serum Institut, Artillerivej 5, DK-2300 Copenhagen, Denmark

Tel +4551955159

Email LISC@ssi.dk
Purpose: Little is known about the prognosis for school performance among children with all-cause infantile hydrocephalus (IHC). Using detailed educational data, we investigated the school performance for IHC patients compared to other children in Denmark.

Patients and methods: We conducted a population-based cohort study of all live-born children in Denmark (1977-2015) based on data from the Danish national health registers and the Danish educational register. The cumulative chance of completing school at age 18 years was estimated using the Aalen-Johansen estimator. The relative risks presented as ORs for not completing school, obtaining grades, or obtaining a grade point average below the national mean value were estimated using a logistic regression model.

Results: The cohort included 2,381,413 children, and of these, 2,573 were diagnosed with IHC. A total of $86 \%$ of IHC children completed compulsory school compared to $96 \%$ among other children; only $62 \%$ of IHC children who completed school received marks vs $96 \%$ among other children. Mediation analyses indicated that one-third of these poorer performances in IHC children could be attributable to their higher prevalence of epilepsy, spasticity, visual disturbances, autism, and attention-deficit hyperactivity disorder. Completion rates were similar for isolated and non-isolated hydrocephalus, and did not vary by age at diagnosis or number of surgeries. Of the children with isolated IHC, $73 \%$ obtained grades vs $58 \%$ of the children with non-isolated IHC. Poorer school performance in IHC children was also observed when considering age at school start, grade point average, and completion of further education.

Conclusion: The poorer school performance among IHC children is particularly reflected by the larger proportion not obtaining grades compared to other children. However, the performance of the IHC children obtaining grades is comparable to that of other children.

Keywords: pediatric hydrocephalus, long-term outcome, prognosis, school performance

\section{Plain language summary}

Hydrocephalus is a condition with accumulation of cerebrospinal fluid within the cavities of the brain. The fluid causes pressure on the surrounding brain, which affects brain development. It is often treated surgically, usually with a shunt that drains the fluid from the brain, but the treatment can be complicated.

So how does the future look for infants diagnosed with hydrocephalus?

We wanted to provide information for the parents who naturally ask this question. One way to describe the overall function of a child is to evaluate the school attendance and performance. As for later function in society, successful school attendance requires not only intelligence but also social skills and physical health. We therefore investigated school performance in children with hydrocephalus compared to other children in Denmark. 
To do so, we used the Danish national registers, which hold detailed health care and educational information on the entire population of Denmark. Of the 2,573 children diagnosed with hydrocephalus, $86 \%$ completed compulsory school as compared to $96 \%$ of the $2,378,840$ other children. Although many children with hydrocephalus completed school, only $62 \%$ of them performed well enough to achieve grades upon completion. Grades were given on a scale from -3 to 12 , and the mean grade point average (GPA) was 5.39 for the hydrocephalus children achieving grades and 6.39 for other children.

Children with hydrocephalus show an overall poor school performance when compared to other children. However, the GPA among the $62 \%$ who did receive grades is comparable to the GPA of other children.

\section{Introduction}

Hydrocephalus is characterized by accumulation of cerebrospinal fluid in the cerebral ventricles. Infantile hydrocephalus (IHC) can be caused by congenital malformations or acquired from cerebral hemorrhage, tumor, traumas, or infections in utero or during infancy. In general, structural brain damage, both due to the underlying causes and hydrocephalus itself, is very common and typically causes cerebral palsy, visual problems, behavioral disorders, and cognitive impairment, but the extent is highly variable. ${ }^{1-3}$ Previous studies have investigated the intelligence quotient (IQ) among children with hydrocephalus and, as expected, generally observed lower IQ scores. ${ }^{4-7}$ However, Hoppe-Hirsch et $\mathrm{al}^{2}$ noted that also hydrocephalic children with normal IQ showed a tendency to be functioning poorly at school, because psychological/behavioral disorders affected their performance negatively. Thus, school performance may be a better end point than IQ scores for functional levels of these children, and it is also a relatable measurement for the parents.

Previous studies are restricted to evaluations of school attendance and the amount of special needs education for the wide-ranging group of pediatric hydrocephalus patients, ${ }^{2,4,8-10}$ whereas no studies could be found evaluating the actual school performances specifically for children diagnosed with hydrocephalus during infancy.

Using the detailed educational data and health information from the virtually complete Danish registers, we were able to investigate school performance specifically for IHC children in a nationwide cohort with almost complete longterm follow-up. The outcomes included timing of school start and completion, achievements of grades, level of GPAs, and completion of further education in IHC children vs other children in Denmark, and we were able to account for clinical factors such as prematurity, etiology, number of surgeries, and comorbidities including epilepsy, spasticity, visual disturbances, autism, and attention-deficit hyperactivity disorder (ADHD).

\section{Materials and methods Data sources}

Since 1968, the Danish Civil Registration System has recorded information on date of birth, kinship, place of residence, and vital status for all citizens in Denmark. ${ }^{11}$ Every individual is assigned a personal identification number (PIN) used in all of the national registers, which enabled cross linkage of information between the registers used in this study. ${ }^{11-14}$

\section{Study cohort}

The cohort for this study comprised all 2,381,413 live-born children in Denmark between January 1, 1977 and July 29, 2015. Children were identified in the Danish Medical Birth Register, which holds information on the birth characteristics of the children born in Denmark, including gestational age and weight. ${ }^{12}$ Preterm birth was defined as birth before week 37 and "small-for-gestational-age" (SGA) as the lowest 10 -percentile weight for each gestational week.

\section{Cases of IHC}

Cases of IHC were cohort members who received a diagnosis of hydrocephalus within the first 2 years of life. They were identified in the Danish National Patient Register, which holds information on all hospital discharge diagnoses and surgeries since 1977, and since 1995 also outpatient and emergency room contacts. Information is recorded by ICD (International Classification of Diseases) codes (ICD 8th edition until 1995 and ICD 10th edition hereafter). ICD codes used in this study are listed in Table S1.

We subdivided the IHC children into the following subgroups, according to the following underlying etiologies: cerebral tumor (diagnosed before age 5 years), spina bifida, Arnold Chiari malformation, perinatal intraventricular hemorrhage, Dandy-Walker syndrome, infection or head trauma, and chromosomal abnormalities/other congenital malformation within or outside the central nervous system. All together, these subtypes of IHC were referred to as nonisolated IHC, whereas IHC was considered to be isolated if none of the abovementioned etiologies applied. When no record of surgical treatment existed for a case of isolated IHC, a minimum of two hospital contacts with a hydrocephalus diagnosis was required to ensure this reflected a true case of IHC. Furthermore, complications to IHC, including epilepsy, visual disturbances, cerebral palsy, autism, and ADHD as well 
as records of surgical treatment for IHC, were also identified in the Danish National Patient Register.

\section{Educational data}

Compulsory schooling in Denmark comprises 1 year of preschool followed by 9 years of schooling, corresponding to primary and lower secondary school. Children normally start to school in August the year they turn 6 years old. All public and private schools in Denmark report administrative data to Statistics Denmark, which generates the nationwide Student Register. The content of the Student Register has expanded over the years, meaning it holds information on completion of compulsory school from 1973 and from 2007 holds information on each completed school year. Since 2002, schools also report preliminary and examination marks for the final year of school. Figure 1 provides an overview of the availability of data for each end point throughout follow-up. Marks are given on a scale ranging from -3 to 12 with a minimal sufficiency at 2 . Furthermore, the register holds information on special needs education from 2011.

Information on further schooling was retrieved from the Population's Education Register, which holds annually recorded information on the highest achieved education for every individual. We divided youth education programs into vocational training and upper secondary school (preparation for higher education).

\section{Statistical analyses}

We estimated cumulative mortality using the Kaplan-Meier estimator, following IHC children from diagnosis and other children from birth, to avoid immortal time bias. For cohort members, who were alive on August 1 the year they turned 4 years old, we estimated time to school start and time to completion of compulsory school using the Aalen-Johansen estimator, taking into account the competing risk of death. The analyses were performed using a discrete time axis with cut points on August 1st or June 1st, respectively, for school start or school completion, and the age the cohort member would reach in the considered year. Cohort members who emigrated/died were censored from the date of emigration/ death. We tested the difference between the estimate for IHC patients and other children using Wald test, assuming the estimate for other children represented the true value for the population.

ORs for school attendance and performance for IHC patients as compared to other children were estimated using a logistic regression model. Analyses were adjusted for sex, birth year, birth month, preterm birth, and SGA. Effects of age at diagnosis, etiology groups, and isolated vs non-isolated IHC on school attendance and performance were evaluated using likelihood ratio tests. Treatment modality (number of surgeries) was evaluated as a trend where the following values were, respectively, considered: $0,1.5$, and the median value for the group with three or more surgeries.

To clarify how much of the observed effect could be explained by complications to IHC (epilepsy, visual disturbances, cerebral palsy, autism, and ADHD), mediation analyses were performed on all four outcomes (see Supplementary material for description of method).

All CIs were 95\% Wald CIs.

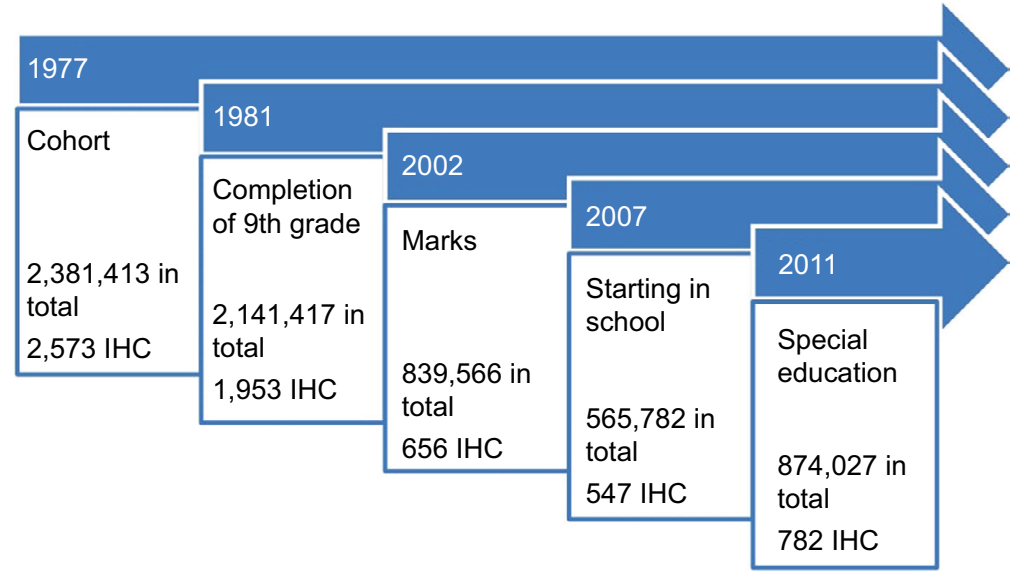

Figure I Overview of the availability of data for each end point throughout follow-up.

Note: Numbers refer to cohort members eligible for analyses.

Abbreviation: IHC, infantile hydrocephalus. 


\section{Ethics}

The study was approved by the Danish Data Protection Agency. According to Danish law, ethical approval is not required for register-based studies.

\section{Results}

Of the 2,381,413 live-born children in the cohort, 2,573 children were diagnosed with IHC, corresponding to 1.08 in 1,000 live-born children. A total of $61.8 \%$ of the IHC patients were males and $38.2 \%$ females, compared to $51.3 \%$ males and $48.7 \%$ females among other children. Characteristics for the IHC children are presented in Table 1 . The cumulative mortality at 4 years of age was $20.0 \%$ for the IHC children as compared to $0.7 \%$ among other children, and increased to $24.9 \%$ for IHC children and $1.0 \%$ for other children at 20 years of age.

Table 2 shows ORs for the outcomes "not starting in school on time", "not completing school", "not receiving marks in 9th grade", "having a GPA below that of other children", and "not completing a youth education program".

\section{School attendance}

Of the cohort members who were alive at school start during the years $2007-2015$, only $69.5 \%$ of IHC patients started on time as compared to $90.4 \%$ of other children. The difference leveled out 1 year later, where $96.8 \%$ of the IHC patients and

Table I Characteristics of children with infantile hydrocephalus

\begin{tabular}{|l|l|}
\hline Characteristics & No of patients (\%) \\
\hline All & $2,573(100)$ \\
Sex & \\
Male & $1,589(61.8)$ \\
Female & $984(38.2)$ \\
Age at diagnosis & \\
0-3 months & $1,409(54.8)$ \\
3-6 months & $401(15.6)$ \\
6-I2 months & $494(19.2)$ \\
I2-24 months & $269(10.5)$ \\
Treatment modality \\
Conservative & \\
I-2 surgeries & $1,473(57.3)$ \\
3+ surgeries & $441(17.1)$ \\
Etiology & $659(25.6)$ \\
Arnold Chiari malformation & \\
Dandy-Walker & $39(1.5)$ \\
Perinatal intraventricular hemorrhage & $75(2.9)$ \\
Spina bifida & $382(14.9)$ \\
Syndromic & $209(8.1)$ \\
Infection or trauma & $1,106(43.0)$ \\
Tumor & $87(3.4)$ \\
Isolated & $101(3.9)$ \\
\hline
\end{tabular}

Note: at age 4 years.
$98.6 \%$ of other children had started school. The cumulative proportion of cohort members who completed compulsory school (9th grade) is presented in Figure 2 (data available from 1981 to 2015). As shown, 54.9\% of the IHC patients finished school on time (at age 16 years), as compared to $82.7 \%$ of other children. Among IHC patients, $13.6 \%$ did not complete compulsory school compared to $4.3 \%$ of other children (at age 18 years), resulting in an OR of 3.38 for not completing school (Table 2). During 2011-2014, where information on special needs education was available, $46.2 \%$ of the IHC children attending compulsory school received special needs education as compared to $5.9 \%$ of other children.

\section{Marks in 9th grade}

The proportion of children finishing receiving 9th grade marks and the proportion obtaining a GPA above average are presented in Table 3. Marks were obtained by $61.9 \%$ of IHC patients and by $95.6 \%$ of other children. The OR for not receiving marks in IHC patients compared to other children was 10.57 (Table 2). The distribution of GPAs in cohort members receiving grades is presented in Figure 3. A mean GPA of 5.39 was observed for IHC children, as compared to 6.39 for other cohort members. Considering 6.39 to be the average GPA for the population, $34.2 \%$ of IHC patients and $51.7 \%$ of other children with marks available obtained a GPA above average. The OR for receiving a GPA below

Table 2 OR for not starting, not completing school, not achieving marks, getting a grade point average $<6.39$, and not getting a youth education for IHC children as compared to other children

\begin{tabular}{|c|c|c|}
\hline End points & Children $^{a}$ & $\begin{array}{l}\text { Adjusted OR } \\
(95 \% \mathrm{CI})^{\mathrm{b}}\end{array}$ \\
\hline \multicolumn{3}{|c|}{ Not starting school on time } \\
\hline Other children & 433,212 & I (ref) \\
\hline IHC & 403 & $4.19(3.30-5.33)$ \\
\hline \multicolumn{3}{|c|}{ Not completing school ${ }^{\mathrm{c}}$} \\
\hline Other children & $\mathrm{I}, \mathrm{II}, 346$ & I (ref) \\
\hline $\mathrm{IHC}$ & 995 & $3.38(2.84-4.04)$ \\
\hline \multicolumn{3}{|c|}{ Not obtaining marks } \\
\hline Other children & 708,903 & I (ref) \\
\hline $\mathrm{IHC}$ & 544 & $10.57(8.84-12.64)$ \\
\hline \multicolumn{3}{|c|}{ Grade point average $<6.39$} \\
\hline Other children & 678,134 & I (ref) \\
\hline IHC & 340 & $1.77(\mathrm{I} .4 \mathrm{I}-2.23)$ \\
\hline \multicolumn{3}{|c|}{ Not getting youth education ${ }^{d}$} \\
\hline Other children & 571,885 & $\mathrm{I}(\mathrm{ref})$ \\
\hline $\mathrm{IHC}$ & 481 & $3.74(3.13-4.49)$ \\
\hline
\end{tabular}

Notes: anly children with follow-up to the relevant time point and with information on covariates were included; ' $O R$ adjusted for birth month, birth year, sex, preterm birth, and SGA; 'by 18 years of age; 'by 25 years of age.

Abbreviations: IHC, infantile hydrocephalus; SGA, small-for-gestational-age; ref, reference. 


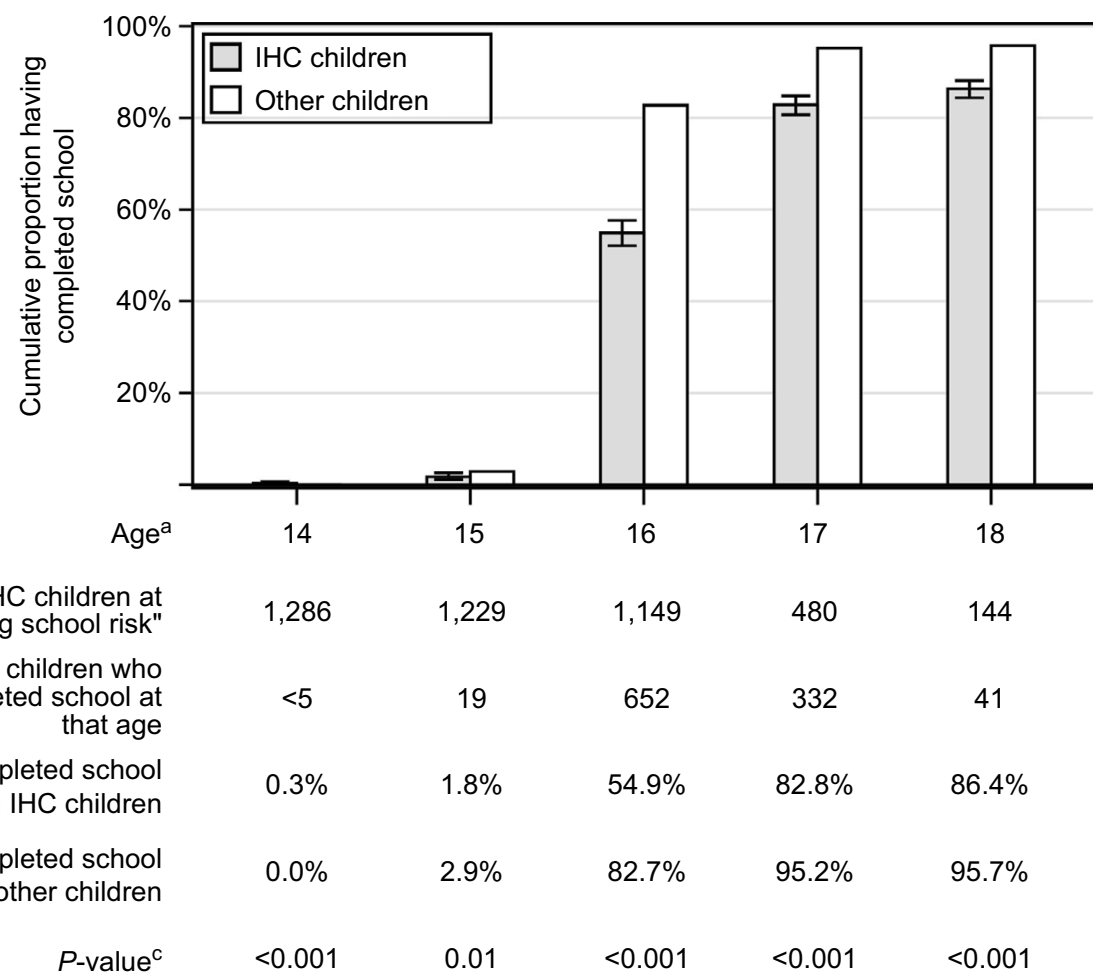

Figure 2 Cumulative proportion that completed school at a given age in IHC and non-IHC children.

Notes: aAll children were assumed to have birthday on the June I (hypothetical date for end of school). ${ }^{\text {bThe }} \mathrm{P}$-values were calculated considering the cumulative proportion for other children as a given value. Data not shown: at age 19 years, there were $100 \mathrm{IHC}$ children at risk of completing school and $<3$ completed school; at age 20 years, the remaining IHC children had still not completed school.

Abbreviations: IHC, infantile hydrocephalus; $\mathrm{CP}$, cumulative proportion.

Table 3 Proportion of children completing 9th grade, \% with grades, and \% with GPA $>6.39$ in IHC children and other children (among children with marks available)

\begin{tabular}{|l|l|l|l|l|}
\hline $\begin{array}{l}\text { Cohort } \\
\text { members }\end{array}$ & $\begin{array}{l}\text { No of children completing } \\
\text { 9th grade }\end{array}$ & $\begin{array}{l}\text { \% with } \\
\text { grades }\end{array}$ & $\begin{array}{l}\text { \% (of those with grades) } \\
\text { with GPA >6.39 }\end{array}$ & $\begin{array}{l}\text { \% (of all children) with } \\
\text { GPA >6.39 }\end{array}$ \\
\hline Other children & 838,910 & 95.6 & 51.7 & 49.4 \\
\hline $\mathrm{IHC}$ & 656 & 61.9 & 34.2 & 21.2 \\
\hline
\end{tabular}

Abbreviations: GPA, grade point average; IHC, infantile hydrocephalus.

average in IHC patients compared with other children was 1.77 (Table 2). Among IHC children, boys and girls seemed to have virtually equal school performances (mean GPA females: 5.38, mean GPA males: 5.39, $P$-value for difference=0.97), whereas for other children, girls had a higher GPA than boys (mean GPA females: 6.75, mean GPA males: $6.04, P$-value for difference $<0.0001)$.

The observed relatively small differences in mean GPAs are most likely confounded by the fact that a high proportion of IHC children did not obtain grades. For a more fair comparison, we compared the median GPAs, when assuming that children without grades would have received a mean GPA below the median. The median GPA was then 3.26 for IHC children as compared to 6.35 for other children, and only $21.2 \%$ of IHC patients as compared to $49.4 \%$ of other children had a GPA above average in non-IHC children.

\section{Youth education programs}

Among the cohort members who completed compulsory school, $46.4 \%$ of IHC children and $78.6 \%$ of other children also completed a youth education program. As presented in Table 2, the risk of not completing a youth education program by age 25 years was 3.74 for IHC children as compared to other children. The youth education programs included upper secondary school, which $49.8 \%$ of IHC children as compared to $65.1 \%$ of other children attended, and vocational training programs, which $50.2 \%$ of IHC children and $34.9 \%$ of other children attended. 


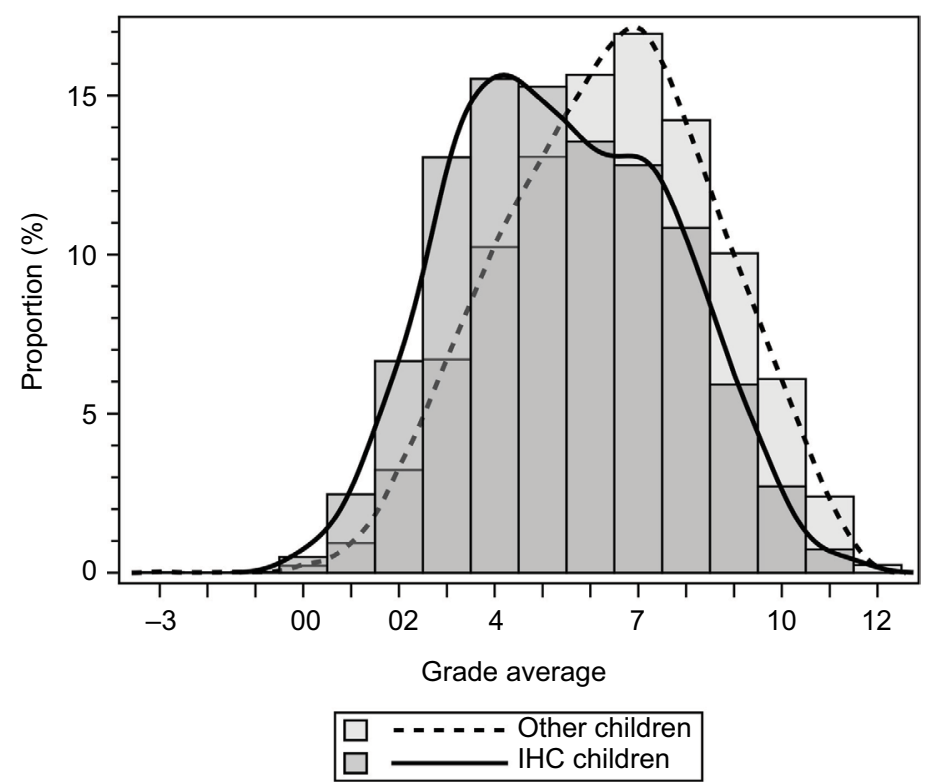

Figure 3 Distribution of grades in 9th grade for IHC children compared to other children.

Note: Density lines for the distribution of GPAs for children with IHC (drawn line) and other children (dotted line).

Abbreviations: IHC, infantile hydrocephalus; GPA, grade point average.

\section{Treatment modality, age at diagnosis, and etiology}

We investigated school attendance and school performance according to treatment modality, age at diagnosis, and etiology of hydrocephalus. The ORs for each outcome are presented in Table S2.

We observed an increased risk of not starting in school on time with increasing number of surgeries in IHC children $(P<0.001)$. In contrast, we observed no association with completion of school or marks. We did not observe associations between age at diagnosis and any of the outcomes.

Due to the limited number of patients in each etiological subgroup, we investigated the differences between children with isolated vs non-isolated IHC. The proportions of the two groups reaching the different end points are presented in Table 4 (ORs are presented in Table S2 and distribution of grades in Figure S1). Children with isolated IHC tended to perform better on all end points. These differences were significant for the chance of obtaining marks in 9th grade, where $73.2 \%$ of isolated IHC children obtained marks as compared to $58.6 \%$ of the children with non-isolated IHC $(P=0.005)$, and for the chance of completing a youth education $(59.1 \%$ vs $41.9 \%[P<0.001])$. The significantly higher risk of a GPA below average among children with non-isolated IHC was imputable to children with hydrocephalus secondary to spina bifida; when excluding these children from the analysis, the difference was no longer significant.
Table 4 Proportion not starting, not completing school, not achieving grades, getting a grade point average below that of other children, and not getting further education in children with isolated and non-isolated IHC

\begin{tabular}{|l|l|l|}
\hline End points & Children $^{\mathrm{a}}$ & $\begin{array}{l}\text { Proportion } \\
\text { reaching } \\
\text { the end point }\end{array}$ \\
\hline Not starting school on time & & \\
\hline Isolated IHC & 128 & $73.1 \%$ \\
\hline Non-isolated IHC & 419 & $68.3 \%$ \\
\hline Not completing school & & \\
\hline Isolated IHC & 290 & $87.7 \%$ \\
\hline Non-isolated IHC & 996 & $86.0 \%$ \\
\hline Not obtaining marks & & \\
\hline Isolated IHC & 149 & $73.2 \%$ \\
\hline Non-isolated IHC & 507 & $58.6 \%$ \\
\hline Grade point average $<6.39$ & & \\
\hline Isolated IHC & 109 & $39.5 \%$ \\
\hline Non-isolated IHC & 297 & $32.3 \%$ \\
\hline Not getting youth education & & \\
\hline Isolated IHC & 149 & $59.1 \%$ \\
\hline Non-isolated IHC & 427 & $41.9 \%$ \\
\hline Notes Onlychidrewth & \\
\hline
\end{tabular}

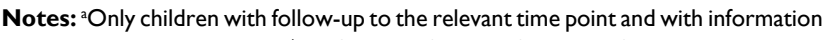
on covariates were included; bat 18 years of age; 'at 25 years of age. Abbreviation: IHC, infantile hydrocephalus.

\section{Effects of epilepsy, visual disturbances, cerebral palsy, autism, and ADHD}

We performed mediation analyses to investigate whether epilepsy, visual disturbances, cerebral palsy, autism, and 
ADHD - all considered complications to IHC - mediated any of the observed effect on the end points. The estimated indirect effects caused by the mediators are presented in Table 5. The mediation analysis suggests that $38.0 \%$ of the increased risk of not completing school in IHC children (OR 3.38) was due to complications related to IHC. For the increased risk of not obtaining marks upon completion of compulsory school (OR 10.57), 34.4\% was mediated by complications to IHC. For the increased risk of having a GPA below that of other children (OR 1.77), 18.9\% was mediated by complications to IHC. The complications to IHC mediated $43.5 \%$ of the increased risk (OR 3.74) of not completing a youth education.

\section{Discussion}

In a population-based cohort with almost 2.5 million individuals, we investigated the school performance in children diagnosed with IHC compared to others. While most IHC children started in school and on average only slightly delayed compared to other children, $13.6 \%$ did not complete compulsory school, as compared to $4.3 \%$ of other children. Among the IHC children who completed school, 61.9\% received marks compared to $96 \%$ of other children and with a GPA of 5.39, as compared to 6.39 for other children. Upon completion of compulsory school, $46.4 \%$ of IHC children completed a youth education program compared to $78.6 \%$ of other children. We observed that complications related to IHC explained $18 \%-43 \%$ of the risks for poorer school performance. Contra-intuitively, their performance did not seem to be influenced by the number of surgeries or age at diagnosis.

Receiving education is mandatory by law in Denmark, which correlates with the observation that only $3.2 \%$ of IHC children had not started school by the age of 7 years. In previous studies, it has been reported that between $6 \%$ and $9 \%$ of children with IHC were not able to attend school. ${ }^{2,8,10}$

Table 5 Estimated proportion of the observed effect of infantile hydrocephalus on not starting, not completing school, not achieving marks, getting a grade point average $<6.39$, and not getting a youth education due to epilepsy, cerebral palsy, visual disturbances, autism, and ADHD

\begin{tabular}{|c|c|}
\hline End points & $\begin{array}{l}\text { Proportion of effect due } \\
\text { to complications }\end{array}$ \\
\hline Not starting school on time & $22.3 \%$ \\
\hline Not completing school ${ }^{b}$ & $38.0 \%$ \\
\hline Not obtaining marks & $34.4 \%$ \\
\hline Grade point average $<6.39$ & $18.9 \%$ \\
\hline Not getting youth education ${ }^{c}$ & $43.5 \%$ \\
\hline
\end{tabular}

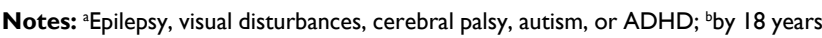
of age; 'by 25 years of age.

Abbreviation: ADHD, attention deficit hyperactivity disorder.
However, none of these studies reported whether any schooling was attempted, which most likely explains the difference. In accordance with other studies, ${ }^{2,9}$ we observed that IHC children tended to be 1 or 2 years behind their peers, and a large proportion of IHC children were not able to complete compulsory school. Nevertheless, our study reveals that a high proportion of IHC children starts and finishes compulsory school.

Only two-thirds of those who completed 9th grade actually achieved marks. Receiving final marks is mandatory and even pupils with physical handicaps, eg, blindness, are not exempted from the standardized tests in 9th grade, which are modified for such cases. Thus, the most plausible explanation for the lower proportion achieving grades is that some IHC children were too cognitively challenged to take the tests. This proportion without grades is an important notion when interpreting the surprisingly small difference in the GPAs between IHC children and other children. As IHC children receiving marks comprise a selected group, the true underlying difference in intellectual skills between IHC children and other children is larger than reflected by the observed difference in GPAs.

Surprisingly, we did not observe that number of surgeries was related with a poorer school performance (with the exception of timing of school start). This is an interesting finding, as one would intuitively expect multiple surgeries to have a negative effect on school performance, as is seen for IQ, ${ }^{15}$ which is either explained by an association with more severe disease or perhaps as a result of the surgeries themselves. Previous studies have suggested that an underlying pathology such as tumor, infection, or intraventricular hemorrhage is correlated with poor outcome. ${ }^{2,4,9}$ In accordance with this, we observed a tendency toward poorer performance in children with non-isolated IHC compared to isolated IHC. Also, subsequent concurrent disease including epilepsy, cerebral palsy, visual disturbances, autism, and ADHD influenced the performance negatively. Nevertheless, neither the underlying pathology nor concurrent diseases fully explained the poorer school performance that we observed in IHC children.

A considerable part of the poor school performance in IHC children must be attributable to low IQ scores., ${ }^{416}$ However, even IHC children with normal IQ tend to perform inferior in school and function poorly in society, ${ }^{2,6,17}$ whereas Preuss et al pointed out that school performance was correlated with social and professional performance in adult life. ${ }^{10}$ Social and behavioral problems seem to be of importance for school performance, ${ }^{2,3}$ but may have a tendency to be underdiagnosed due to more obvious disabilities. In one study, Lindquist et al observed that seven out of the $67 \mathrm{IHC}$ 
patients had undiagnosed autism and that $12 \%$ of the patients with normal IQ had behavioral problems. ${ }^{3}$ While IQ classification has the advantage of being a standardized method and is easily tested, our data offer a possibility to give an informed and more precise prognosis for infants with IHC, which is comprehensible for their parents.

This population-based study is, to our knowledge, the first study investigating school performance in compulsory school specifically by marks. The unique registers allowed us to construct a non-selected nationwide cohort with close to complete follow-up. In comparison, all previous studies on schooling of IHC children are based on single center data except from Gupta et al, who relied on American neurosurgeons to handout questionnaires to patients. ${ }^{18}$ The educational register covers a wide range of educational data, and the tests taken upon completion of compulsory school are standardized and used nationally in virtually all private and public schools. Since health care is easily accessible and free, and reporting discharge diagnoses on all hospital contacts is mandatory by law, the registration of hydrocephalus is considered to be nearly complete. An important limitation of our study was the variable availability of data during follow-up due to expansion of the educational registers over the years (Figure 1), meaning the cohorts for each outcome are different. Since etiology and treatment have not changed much over the years, and since we adjusted for time period, we do believe that the cohorts are comparable.

\section{Conclusion}

We found that most children with IHC attend and to a high degree also finish compulsory school. However, their performance, judged by their ability to obtain grades and by the level of their grades, is significantly poorer than for other children. This pattern was most pronounced for non-isolated IHC children, but in general not influenced by age at diagnosis and number of surgeries. However, the performance of the IHC children obtaining grades is comparable to that of other children.

\section{Acknowledgments}

The study was supported by scholarships from the Novo Nordisk Foundation and The A.P. Møller Foundation for the Advancement of Medical Science.

\section{Author contributions}

Dr Schmidt participated in designing the study; she coordinated and supervised data collection, interacted with the statisticians regarding the analyses, and drafted and revised the manuscript. Ms Corn carried out the statistical analyses, supervised by Mr Wohlfahrt, and both critically reviewed and revised the manuscript. Dr Melbye assisted in designing the study, and critically reviewed and revised the manuscript. Dr Munch conceptualized and designed the study, and critically reviewed and revised the manuscript. All authors gave final approval of the manuscript version to be published and agree to be accountable for all aspects of the work.

\section{Disclosure}

The authors report no conflicts of interest in this work.

\section{References}

1. Vinchon M, Rekate H, Kulkarni AV. Pediatric hydrocephalus outcomes: a review. Fluids Barriers CNS. 2012;9(1):18.

2. Hoppe-Hirsch E, Laroussinie F, Brunet L, et al. Late outcome of the surgical treatment of hydrocephalus. Childs Nerv Syst. 1998;14(3):97-99.

3. Lindquist B, Carlsson G, Persson EK, Uvebrant P. Behavioural problems and autism in children with hydrocephalus : a population-based study. Eur Child Adolesc Psychiatry. 2006;15(4):214-219.

4. Heinsbergen I, Rotteveel J, Roeleveld N, Grotenhuis A. Outcome in shunted hydrocephalic children. Eur J Paediatr Neurol. 2002;6(2):99-107.

5. Lindquist B, Persson EK, Fernell E, Uvebrant P. Very long-term followup of cognitive function in adults treated in infancy for hydrocephalus. Childs Nerv Syst. 2011;27(4):597-601.

6. Lacy M, Pyykkonen BA, Hunter SJ, et al. Intellectual functioning in children with early shunted posthemorrhagic hydrocephalus. Pediatr Neurosurg. 2008;44(5):376-381.

7. Dalen K, Bruarøy S, Wentzel-Larsen T, Laegreid LM. Intelligence in children with hydrocephalus, aged 4-15 years: a population-based, controlled study. Neuropediatrics. 2008;39(3):146-150.

8. Vinchon M, Baroncini M, Delestret I. Adult outcome of pediatric hydrocephalus. Childs Nerv Syst. 2012;28(6):847-854.

9. Paulsen AH, Lundar T, Lindegaard KF. Pediatric hydrocephalus: 40-year outcomes in 128 hydrocephalic patients treated with shunts during childhood. Assessment of surgical outcome, work participation, and health-related quality of life. J Neurosurg Pediatr. 2015;16(6):633-641.

10. Preuss M, Kutscher A, Wachowiak R, et al. Adult long-term outcome of patients after congenital hydrocephalus shunt therapy. Childs Nerv Syst. 2015;31(1):49-56.

11. Pedersen CB. The Danish Civil Registration System. Scand J Public Health. 2011;39(7 Suppl):22-25.

12. Munch TN, Rostgaard K, Rasmussen ML, Wohlfahrt J, Juhler M, Melbye M. Familial aggregation of congenital hydrocephalus in a nationwide cohort. Brain. 2012;135(Pt 8):2409-2415.

13. Jensen VM, Rasmussen AW. Danish Education Registers. Scand J Public Health. 2011;39(7):91-94.

14. Schmidt M, Schmidt SA, Sandegaard JL, Ehrenstein V, Pedersen L, Sørensen HT. The Danish National Patient Registry: a review of content, data quality, and research potential. Clin Epidemiol. 2015;7:449-490.

15. Arrington CN, Ware AL, Ahmed Y, Kulesz PA, Dennis M, Fletcher JM. Are Shunt Revisions Associated with IQ in Congenital Hydrocephalus? A Meta -Analysis. Neuropsychol Rev. 2016;26(4):329-339.

16. Lumenta CB, Skotarczak U. Long-term follow-up in 233 patients with congenital hydrocephalus. Childs Nerv Syst. 1995;11(3):173-175.

17. Kokkonen J, Serlo W, Saukkonen AL, Juolasmaa A. Long-term prognosis for children with shunted hydrocephalus. Childs Nerv Syst. 1994;10(6):384-387.

18. Gupta N, Park J, Solomon C, Kranz DA, Wrensch M, Wu YW. Longterm outcomes in patients with treated childhood hydrocephalus. $J$ Neurosurg. 2007;106(5 Suppl):334-339. 


\section{Supplementary materials \\ Methods for mediation analysis}

Mediation analysis was performed for all five outcomes to clarify how much of the observed effect could be explained by complications to infantile hydrocephalus (IHC) (epilepsy, visual disturbances, cerebral palsy, autism, or attention-deficit hyperactivity disorder). The pure direct effect of IHC and the total indirect effect of implications were estimated using the Lange decomposition, ${ }^{1}$ estimating first a mediator model and subsequently a weighted model for the outcome. The mediator was defined as a 16-level variable considering all possible combinations of the four mentioned complications, and the mediator model was fitted by decomposing the joint probability of the 16 levels using chain rule and estimating each of the conditional models separately. The proportion not explained by the four factors was calculated on log-scale, as the ratio between the pure direct effect and total effect. Weights were truncated so much so that the ratio between the total effect estimated with logistic regression and the one obtained from mediation analysis became less than 1.02. Sex, birth year, birth month, preterm birth, and small-for-gestational-age were included in both the mediator and the outcome model.

Table SI Disease diagnoses according to the ICD codes

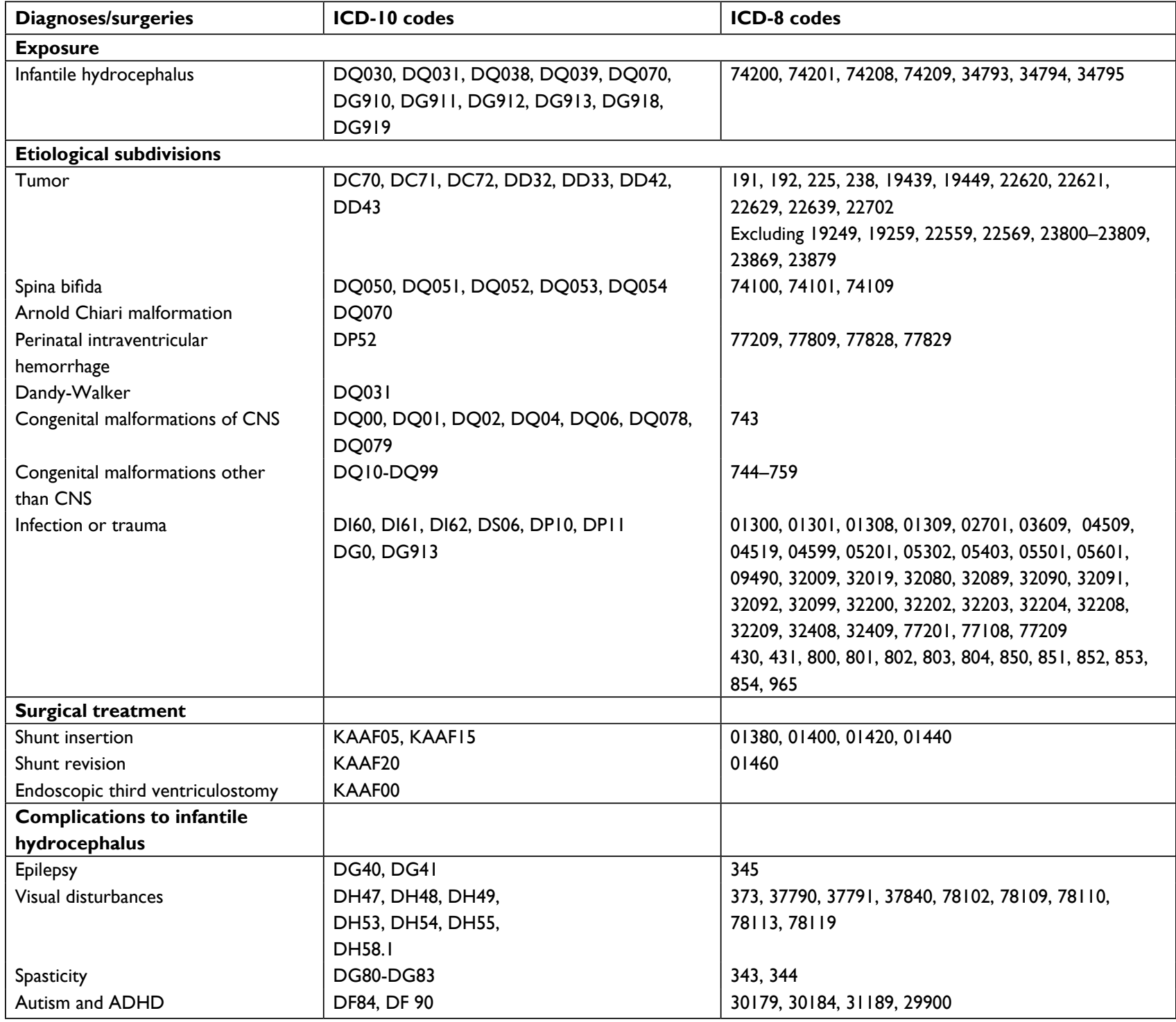

Abbreviations: ADHD, attention-deficit hyperactivity disorder; CNS, central nervous system; ICD-8, International Classification of Diseases 8th edition; ICD-I0: International Classification of Diseases I0th edition. 


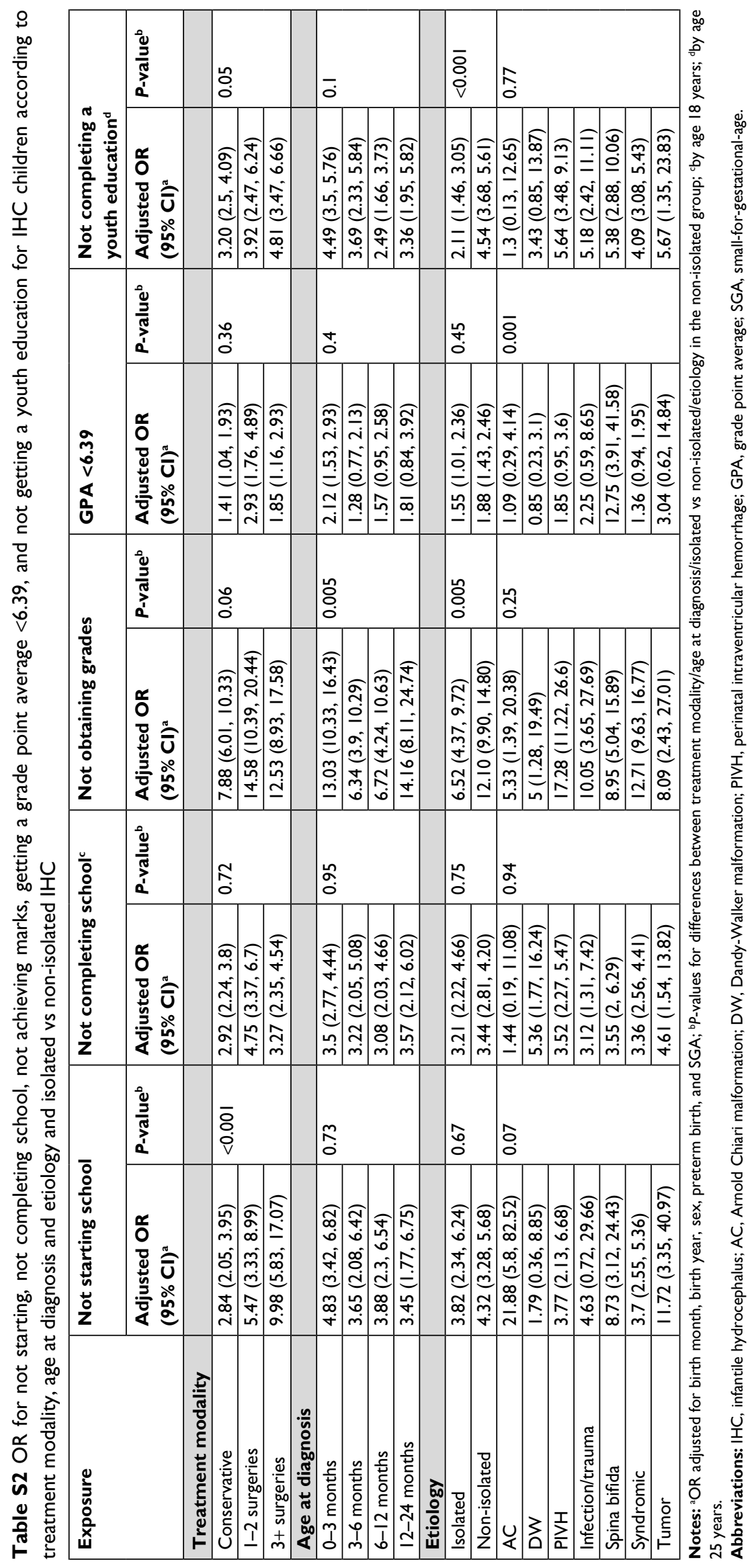



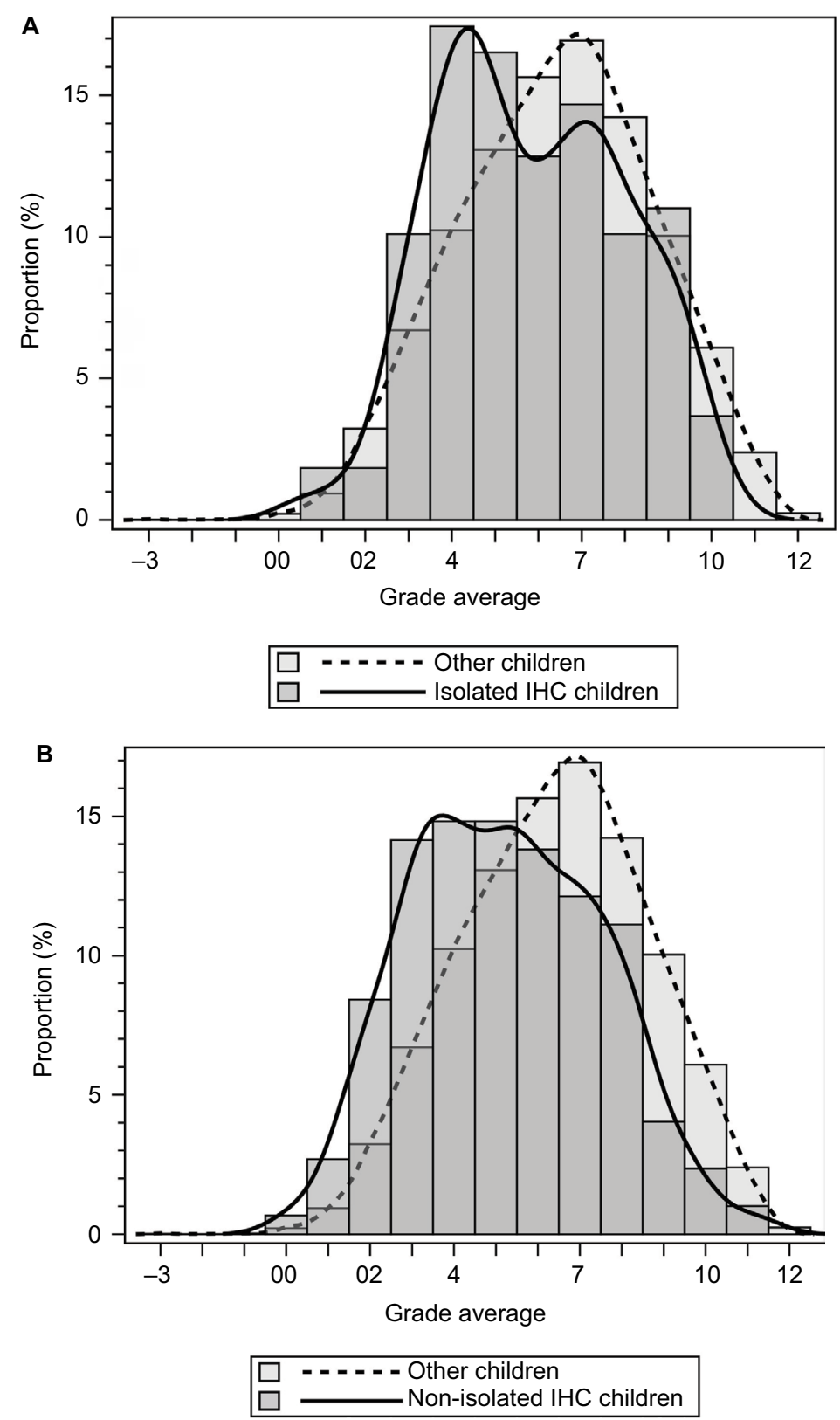

Figure SI Distribution of grade point averages for isolated (A) and non-isolated (B) IHC as compared to other children. Abbreviation: IHC, infantile hydrocephalus.

\section{Reference}

1. Lange T, Vansteelandt S, Bekaert M. A simple unified approach for estimating natural direct and indirect effects. Am J Epidemiol. 2012;176(3):190-195.

\section{Clinical Epidemiology}

\section{Publish your work in this journal}

Clinical Epidemiology is an international, peer-reviewed, open access, online journal focusing on disease and drug epidemiology, identification of risk factors and screening procedures to develop optimal preventative initiatives and programs. Specific topics include: diagnosis, prognosis, treatment, screening, prevention, risk factor modification, systematic reviews, risk and safety of medical interventions, epidemiol-
ogy and biostatistical methods, and evaluation of guidelines, translational medicine, health policies and economic evaluations. The manuscript management system is completely online and includes a very quick and fair peer-review system, which is all easy to use. 